\title{
Cuidados paliativos em favelas no Brasil: Uma revisão integrativa
}

\author{
Palliative care in favelas in Brazil: An integrative review \\ Cuidados paliativos en las favelas de Brasil: Una revisión integradora
}

Recebido: 17/05/2021 | Revisado: 23/05/2021 | Aceito: 25/05/2021 | Publicado: 09/06/2021

\author{
Alexandre Ernesto Silva \\ ORCID: https://orcid.org/0000-0001-9988-144X \\ Universidade Federal de São João Del Rei, Brasil \\ E-mail: alexandresilva@ufsj.edu.br \\ Flávia Borba Paulino Coelho \\ ORCID: https://orcid.org/0000-0003-0295-2673 \\ Universidade Federal de São João Del Rei, Brasil \\ E-mail: flavia.borbac@gmail.com \\ Frederico Mendes Silva Pereira \\ ORCID: https://orcid.org/0000-0003-3724-2743 \\ Universidade Federal de São João Del Rei, Brasil \\ E-mail: fred147159@gmail.com \\ Ismael Carrilho de Castro \\ ORCID: https://orcid.org/0000-0003-3171-7613 \\ Universidade Federal de São João Del Rei, Brasil \\ E-mail: ismaelccastro@gmail.com \\ Larissa Silva Braga \\ ORCID: https://orcid.org/0000-0002-4105-6524 \\ Universidade Federal de São João Del Rei, Brasil \\ E-mail: larissa.sbraga@hotmail.com \\ Moisés Fiúsa Menezes \\ ORCID: https://orcid.org/0000-0002-7373-1205 \\ Universidade Federal de São João Del Rei, Brasil \\ E-mail: moisesfiusa4@gmail.com \\ Patrícia Soares Mesquita \\ ORCID: https://orcid.org/0000-0001-6597-3485 \\ Universidade Federal de São João Del Rei, Brasil \\ E-mail: patriciasoaresmesquita@gmail.com \\ Regis Michel Ribeiro Martins \\ ORCID: https://orcid.org/0000-0002-6124-5760 \\ Universidade Federal de São João Del Rei, Brasil \\ E-mail: regismichel@yahoo.com.br \\ Stefãne Amorim Ribeiro \\ ORCID: https://orcid.org/0000-0001-9702-1913 \\ Faculdade Asa de Brumadinho, Brasil \\ E-mail: stefanearibeiro@yahoo.com.br \\ Thayane Vieira Carvalho \\ ORCID: https://orcid.org/0000-0001-8644-8954 \\ Clínica Auge, Brasil \\ E-mail: thayanevieira100@yahoo.com.br
}

\begin{abstract}
Resumo
Objetivo: Identificar como os Cuidados Paliativos (CPs) são empregados aos moradores de favelas brasileiras. Método: Revisão bibliográfica integrativa, na qual foram analisados artigos publicados entre 2019 e 2020 , nas bases de dados Scielo, Lilacs, Bdenf e Medline (via PubMed). Incluíram-se artigos originais, disponíveis na íntegra e que respeitassem o limite temporal estabelecido. Resultados e discussão: 25 artigos foram selecionados; 19 tratam-se da situação dos CPs no Brasil e 6 abordam especificamente os CPs em comunidades vulneradas, sendo divididos em 2 categorias: "a compreensão do acesso à saúde de moradores de favelas" e "dificuldades de acesso aos CPs em áreas de pobreza". Tais artigos mostram que as necessidades de pessoas elegíveis aos CPS nas favelas são desconhecidas pelos serviços públicos de saúde. Conclusão: Barreiras culturais em torno da morte e do morrer associadas a falta de investimento financeiros e educacionais contribuem para um incipiente desenvolvimento dos cuidados paliativos no Brasil. Ademais, é importante destacar a necessidade da produção de estudos específicos sobre os cuidados paliativos em favelas, para levantar informações que subsidiem ações adequadas na garantia do direito à saúde e bem estar no final de vida para essa população.
\end{abstract}

Palavras-chave: Cuidados paliativos; Áreas de pobreza; Grupos de risco; Atenção primária à saúde. 


\begin{abstract}
Objectives: Identify how palliative care (PC) is offered for residents of brazilian favelas. Methods: This is a bibliographic review of the integrative type, from which were analyzed articles published between the year of 2014 and 2019, in the Scielo, Lilacs, Bdenf and Medline databases (via PubMed). On this study were included original articles that were available in full text and that respected the established time limit. Results and discussion: 25 articles were selected. 19 of them were about the situation of PC in Brazil and 6 of them were about PC in vulnerable communities, and they were divided in two categories: "the understanding of health access for favela's residents" and "difficulties in accessing palliative care in poverty areas". These articles show that the needs of people eligible for PC in favelas are unknown by public health services. Conclusion: Cultural barriers around death and dying associated with lack of financial and educational investments contribute for an incipient development of palliative care in Brazil. In addition, is important to highlight the need of further studies about the availability of this service on poor communities in Brazil to raise information to support the provision of appropriate actions to guarantee the right to health and well-being of life of this population.
\end{abstract}

Keywords: Palliative care; Poverty areas; Risk groups; Primary health care.

\title{
Resumen
}

Objetivo: Identificar cómo se utilizan los Cuidados Paliativos (CP) para los habitantes de los barrios marginales brasileños. Método: Revisión bibliográfica integradora, en la calificación de artículos publicados entre 2019 y 2020 , en las bases de datos Scielo, Lilacs, Bdenf y Medline (vía PubMed). Se incluyeron artículos originales, disponibles íntegramente y respetando el plazo establecido. Resultados y discusión: se seleccionaron 25 artículos; 19 abordan la situación de los PC en Brasil y 6 específicamente abordan los PC en comunidades de acceso vulnerable, divididos en 2 categorías: "comprensión de la salud de los habitantes de tugurios" y "dificultades para acceder a los PC en zonas de pobreza". Estos artículos muestran que los servicios de salud pública desconocen las necesidades de las personas elegibles para la atención primaria de salud en los barrios marginales. Conclusión: Las barreras culturales en torno a la muerte y el morir asociados con la falta de inversión financiera y educativa contribuyen a un desarrollo incipiente de los cuidados paliativos en Brasil. Además, es importante resaltar la necesidad de realizar estudios específicos sobre cuidados paliativos en las favelas, con el fin de recabar información que sustente las acciones necesarias para garantizar el derecho a la salud y el bienestar al final de la vida de esta población.

Palabras clave: Cuidados paliativos; Zonas de pobreza; Grupos de riesgo; Atención primaria a la salud.

\section{Introdução}

A população brasileira, acompanhando uma tendência mundial, está envelhecendo, e esse fenômeno implica em mudanças significativas nas perspectivas em saúde. Com uma maior longevidade, a prevalência de doenças crônicodegenerativas aumenta, e as necessidades de cuidado advindas de muitas delas começam a ter uma maior relevância ${ }^{1}$. É nesse cenário, pois, que surge a importância dos cuidados paliativos $(\mathrm{CP})$ : uma prática que visa melhorar a qualidade de vida de pacientes e seus familiares diante de doenças que ameaçam a continuidade da vida (Nickel, Oliari, Vesco \& Padilha, 2016).

Os Cuidados Paliativos, segundo a Organização Mundial de Saúde (OMS), são definidos como uma abordagem que valoriza a qualidade de vida, dos doentes e suas famílias no enfrentamento dos problemas ocasionados por doenças ameaçadoras à vida, através da prevenção e alívio do sofrimento, identificação precoce, avaliação correta e tratamento da dor e outros problemas de ordem física, psicossocial e espiritual (WHO, 2020).

Ressalta-se que esses cuidados não se restringem apenas aos idosos ou a pacientes com doenças incuráveis, podendo ser expandidos para outras pessoas que também cursam com condições que ameaçam a continuidade da vida (Ribeiro \& Poles, 2019).

No país, o tema dos CP começou a ter visibilidade a partir da detecção de problemas na rede de assistências em saúde, que levou o Ministério da Saúde (MS) a trabalhar em programas e políticas nacionais de humanização ${ }^{3}$, de modo que, na década de 1980, se inicia a implementação dos primeiros serviços de CP no país. Posteriormente, em 1997, criou-se a Associação Brasileira de Cuidados Paliativos (ABCP) e em 2002 o Programa Nacional de Assistência à Dor e Cuidados Paliativos (Nickel, Oliari, Vesco \& Padilha, 2016).

Um levantamento sobre a disponibilidade de serviços de CP no país mostrou que há uma desigualdade considerável na oferta do serviço, visto que se constatou haver uma concentração de mais de $50 \%$ apenas na região sudeste. Também foi 
identificado que a maior parte desse cuidado é feito em hospitais. Ainda assim, menos de $10 \%$ dos hospitais possuem uma equipe capacitada para esse trabalho (Academia Nacional de Cuidados Paliativos, 2018).

O Brasil precisa de investimento maior para conseguir fornecer um acesso adequado aos cuidados paliativos. No país, falta o estabelecimento de muitas questões básicas para a aplicação desse serviço, como a formação e capacitação de profissionais e o fornecimento dos instrumentos necessários para a prática. A situação de precariedade do serviço se agrava ainda mais quando se trata de comunidades vulneradas, termo referente àquelas comunidades inseridas em realidades onde a resultante entre fatores de risco e de proteção aponta para níveis mais elevados de risco ${ }^{5}$. Conforme o último censo do IBGE (Instituto Brasileiro de Geografia e Estatística), de 2010, cerca de 11 milhões de brasileiros habitam 6.329 favelas, sendo que 19,1\% destas estão localizadas no estado do Rio de Janeiro (IBGE, 2010). Além disso, em 2019 havia 5.127 .747 milhões de domicílios ocupados em 13.151 mil aglomerados subnormais no país(IBGE, 2020).

Ademais, as vulnerabilidades são distintas tais quais as comunidades expostas, ou seja, o contexto proporciona proteção e exposições variadas, conferindo diferentes níveis de bem-estar. Nas sociedades capitalistas, essa vulnerabilidade está inserida em maior grau nas populações pobres, e essa parcela populacional enfrenta dificuldades de acesso aos recursos para superação dessas barreiras devido à fragilidade de acesso à renda e infraestrutura (Scott, Prola, Siqueira \& Pereira, 2018; Carmo \& Guizardi, 2018). Nesse contexto, é viável assumir que as favelas brasileiras apresentam a baixa renda como fator agravante ao acesso de seus direitos fundamentais, tornando-as parte fundamental quando se pensa em pessoas em situação de vulnerabilidade.

Portanto, para os moradores de comunidades vulneradas o prejuízo é patente, posto que pessoas nascidas em favelas têm a expectativa de vida diminuída em 13 anos somente por viverem onde vivem, expostas a vulnerabilidades inerentes desse locus, definido pelo Instituto Brasileiro de Geografia e Estatística (IBGE).

Favela, grotão, comunidade, alagado, entre outros, é cada conjunto constituído de no mínimo 51 unidades habitacionais carentes, em sua maioria, de serviços públicos essenciais, estando dispostas, em geral, de forma desordenada e densa ou ocupar terreno de propriedade alheia (pública ou particular) (IBGE, 2010).

Dessa maneira, pessoas inseridas na díade vulnerado/comunidade vulnerada vivem a soma dos prejuízos de sua realidade individual e do contexto social. Essa agregação pode ser notada em moradores de favelas que necessitam de cuidados paliativos, os riscos individuais e coletivos juntos implicam em graus de exposições a riscos de sofrimento mais comprometedores do que a população em geral das favelas, como também pessoas em cuidados paliativos fora desse contexto (Schramm, 2007).

Sendo assim, esta pesquisa objetiva responder a seguinte pergunta: como os cuidados paliativos são ofertados em favelas do Brasil? Para tanto, buscou-se identificar como os CP são empregados aos moradores de favelas brasileiras.

\section{Metodologia}

Em busca de uma abordagem metodológica abrangente que atenda as peculiaridades da investigação dos cuidados paliativos em comunidades vulneradas, a presente pesquisa constitui-se uma revisão bibliográfica integrativa. Desenho de estudo que melhor delineia as produções acadêmicas acerca do tema mediante os diversos níveis de evidência científica, proporcionando um panorama consistente da investigação científica realizada até o presente momento (Souza, Silva \& Carvalho, 2010).

O estudo se constrói em torno das etapas base de uma revisão integrativa. O problema norteador do estudo foi definido como a caracterização dos cuidados paliativos em comunidades vulneradas, com ênfase em favelas do Brasil. Em seguida, para a busca e construção da amostra foram utilizadas: a Biblioteca Virtual em Saúde (BVS), a Scientific Eletronic 
Library Online (Scielo) e a base Medline por meio da National Library of Medicina National Institutes of Health (PubMed). Para tal, os descritores utilizados para refinar a pesquisa foram: cuidados paliativos, áreas de pobreza, grupos de risco, atenção primária à saúde. A coleta aconteceu entre setembro de 2019 a abril de 2020, tendo como critérios de inclusão produções em português e inglês com até 5 anos de publicação, as quais o título e conteúdo abordem o tema definido, os artigos de consulta gratuita e na íntegra nos bancos de dados. Excluiu-se da pesquisa produções que fugiam do contexto definido e que não se enquadraram em nenhum dos 6 níveis de evidência científica.

\section{Resultados e Discussão}

A busca nas bases de dados levando em consideração os critérios de inclusão e exclusão definidos resultou em uma amostra preliminar de 25 artigos. Essas produções foram submetidas à leitura e análise visando detectar o desenho de estudo escolhido, o nível de evidência científica, a área de conhecimento, as variáveis analisadas e a similaridade com a temática proposta. A busca através dos descritores principais "cuidados paliativos" e "áreas de pobreza" não teve êxito, pois não foi encontrada nenhuma produção científica que abordasse especificamente os CP nessas áreas, tratando de favelas no Brasil até o momento da pesquisa. Ao se focar em textos que abordam parcialmente ou que tem similaridade com a proposta foram selecionados 6 estudos considerados adequados, os quais constituíram o objeto de estudo desta revisão.

Dentre os artigos, 4 foram publicados em revistas internacionais em língua inglesa e 3 em revistas nacionais em língua portuguesa. Referente às bases de dados, 2 são provenientes da BVS, 3 da Medline e 2 da Scielo. Os estudos são majoritariamente da área de ciências da saúde (57\%), seguido de enfermagem (28\%) e medicina (15\%). Números que demonstram incipiente interesse científico no que se refere aos cuidados em saúde nas favelas, além da lacuna relativa ao conhecimento dos CP nesses ambientes sociais.

Os desenhos de estudo evidenciam uma situação peculiar nos estudos de CP, um foco expressivo em trabalhos descritivos qualitativos que somam 57\% dos artigos, seguido por estudos opinativos 28\%, sendo apenas $15 \%$ de estudos descritivos não experimentais quantitativos. Ressalta-se que essa pesquisa não conseguiu encontrar nenhum ensaio clínico, tampouco estudos experimentais e caso-controle. Tal fato pode ser compreendido devido à natureza do objeto em questão, uma vez que seria antiético submeter esses pacientes a pesquisas experimentais que possivelmente privariam uma parcela do grupo de estudo de receber o tratamento.

Conforme os parâmetros da Saúde Baseada em Evidência, que define 6 níveis de evidência científica a respeito das produções, nenhum dos estudos encontrados abrangiam os níveis 1 (meta-análises de múltiplos ensaios clínicos controlados e randomizados), 2 (estudos individuais com delineamento experimental) e 3 (estudos quase-experimentais), os quais apresentam estudos com evidências mais concretas e seguras em relação ao objeto estudado. Destaca-se que $71 \%$ dos artigos são de nível 4 que abrangem os estudos descritivos não-experimentais e os com abordagem qualitativa, os outros $29 \%$ tratam-se de produções baseadas em opiniões de especialistas da área. Apesar dos estudos serem de qualidade comprovada, originais e publicados em revistas de renome, eles possuem entre mediana e baixa evidência científica. Portanto, o conhecimento acadêmico referente aos CP em favelas necessita de investimentos de grupos de pesquisa e estudiosos individuais a fim de aumentar a segurança e profundidade do assunto e, por conseguinte, melhorar o cuidado em saúde dessa população.

Após análise dos artigos foram encontradas 6 temáticas de discussão, quais sejam: 1 - Cuidados paliativos no mundo; 2 - Desafios da Atenção Domiciliar (AD) na Atenção Primária à Saúde (APS) no SUS; 3 - Acesso à saúde nas favelas brasileiras; 4 - APS na Rocinha; 5 - CP para os sem teto no Canadá; 6 - Barreiras para o acesso aos CP. As temáticas 2, 3 e 4, que concorrem na categoria "Os CP no mundo e nas Favelas Brasileiras”, foram escolhidas para melhor compreensão de como o acesso à saúde acontece em relação aos moradores de favelas. Essas asserções mostraram que a AD se encontra 
negligenciada no SUS devido à alta demanda e poucas equipes, o acesso e integralidade dos serviços são baixos na Rocinha e que a disparidade social causada pela falta de renda reflete na dificuldade de acesso à saúde nas favelas. Já as temáticas 1 , 5 e 6 apontam para a deficiência de políticas de saúde, necessidade de melhor formação profissional, oferta de serviços e medicamentos, e confluem para a categoria "A complexidade das Favelas brasileiras e os CP". Além disso, também se observou estratégias falhas para garantir CP aos moradores de rua do Canadá, e que muitas pessoas vivendo com doenças crônicas ameaçadoras de suas vidas não recebem CP ou os recebem apenas na fase final de doença. Assim, é possível entender, com a similaridade entre os artigos, que o acesso à saúde, principalmente de CP por pessoas pobres é limitado e que isso é verdade no caso de favelas brasileiras (Huynh, Henrym \& Dosani, 2015; Hawley, 2017).

\section{Os CP no mundo e nas Favelas Brasileiras}

A Organização Mundial de Saúde (OMS), em 2014, no Global Atlas of Palliative Care at the End of Life (Atlas Global de Cuidados Paliativos no Final da Vida), enfatiza que os cuidados paliativos devem ser um direito de todo cidadão, independentemente de sua situação socioeconômica (Huynh, Henrym \& Dosani, 2015). No entanto, a oferta desse serviço no Brasil ainda é muito escassa, diferentemente de outros países que já estão mais avançados nesse quesito.

Em 2010 a revista The Economist publicou o Índice de Qualidade de Morte, que pontuava os países em quatro categorias: ambiente para cuidados básicos de fim de vida, disponibilidade de serviços para esse cuidado, custo e investimento neles e sua qualidade. Foram avaliados indicadores quantitativos, como expectativa de vida, indicadores qualitativos, como o conhecimento da sociedade desse serviço, e indicadores de status, que descrevem a existência ou não de alguma ação ou política nos países. Assim, países como Reino Unido, Austrália e Nova Zelândia tiveram os melhores resultados. Já nos países em desenvolvimento, a pontuação foi menor no quesito de ambientes para cuidados básicos de fim de vida e disponibilidade de serviços. Tal característica pode estar relacionada com a cultura do país em relação à morte e ao morrer ou com a falta de investimento por parte do governo em saúde, principalmente nessa área (Victor, 2016).

No Reino Unido, boa parte das despesas destinadas aos cuidados paliativos vem de uma rede de hospices sem fins lucrativos, o que reduz a demanda de investimentos pelo Serviço Nacional de Saúde. Já na Austrália, os impostos federais são destinados a estados e municípios com a finalidade de investir nesse tipo de serviço, o que reduz a demanda de investimentos pelo Serviço Nacional de Saúde. Além disso, esses dois países, juntamente do México, Nova Zelândia, Polônia, Suíça e Turquia, são os únicos dos 40 países analisados que já tinham implantado políticas nacionais de saúde que contemplem os CP. Portanto, graças a todo esse investimento e preparação que a Austrália, o Reino Unido e a Nova Zelândia continuaram liderando o ranking do Índice de Qualidade de Morte quando realizado novamente em 2015 (Victor, 2016).

É importante destacar que os países com melhores posições globais são aqueles que possuem políticas governamentais sobre cuidados paliativos. No entanto, existem outros pontos analisados. Um deles é o envolvimento social nos CP, que engloba o envolvimento de familiares, voluntários e a própria comunidade, o que reduz os custos relacionados às internações e às admissões em serviços de emergência. Nessa categoria o Brasil teve um ótimo desempenho, sendo classificado em $3^{\circ}$ lugar (Jeanniton, 2016).

Apesar da boa classificação em uma das categorias, o Brasil ainda mostra diversas falhas na implementação dos CP. A exemplo disso, pode-se citar o levantamento publicado pela OMS em 2014 sobre o desenvolvimento desse serviço no mundo. O Brasil foi classificado como 3A, o que significa que o fornecimento dessa modalidade em saúde no país é feito de forma isolada, e seu desenvolvimento recebe pouco apoio, sendo consideravelmente dependente de doações. Essa classificação também demonstra que há pouca disponibilidade de morfina e pouca quantidade de serviços de $\mathrm{CP}$ para o tamanho da população (Academia Nacional de Cuidados Paliativos, 2018). 
Corroborando o resultado da análise da OMS, a pesquisa já citada da The Economist sobre a qualidade de morte em 80 países em 2015, avaliando acesso a opioides, políticas públicas em cuidados paliativos e acesso a esses cuidados no sistema de saúde, mostrou um atraso nesse quesito no Brasil. O país foi classificado em $42^{\circ}$ lugar, estando pior do que países como Uganda e Malásia. Percebe-se, portanto, que para alcançar a plena implementação dessa área no sistema de saúde brasileiro ainda é necessário enfrentar diversos obstáculos, sendo necessária a criação de políticas públicas direcionadas para a correção desses problemas identificados (Academia Nacional de Cuidados Paliativos, 2018).

Além disso, estudo sobre a população brasileira e a oferta de CP mostrou que, em 2000, a projeção média de unidades de internação era de 624, que havia a necessidade de cerca de 4.779 médicos e 8.568 enfermeiros e o número de equipes de atenção básica/atendimento domiciliar necessário para atender à demanda da população era de 1.156. Esse número, em 2040, subiria para 1.521, e a projeção de unidades de internação aumentaria para 821. Ademais, nesse ano seriam necessários 6.274 médicos e 11.294 enfermeiros para os cuidados paliativos. Ainda dentro dessa pesquisa, foi estimado que, em 2.040, 10.267 leitos de hospitais seriam necessários para esse tipo de serviço (Santos, Campos, Barros, Serafim, Klug \& Cruz, 2019). Portanto, observa-se uma necessidade emergente e cada vez maior de investir nessa modalidade de atendimento em saúde.

Um mapeamento realizado pela Academia Nacional de Cuidados Paliativos (ANCP) em 2018, mostrou que haviam 177 serviços de CP no Brasil até agosto daquele ano. A maior parte destes se encontravam na região sudeste (58\%), enquanto a menor parcela no norte do país (3\%). Ainda, evidenciou-se que mais de $50 \%$ deles iniciaram seus serviços a partir do ano de 2010. Destacou-se, também, que 74\% deles funcionavam em hospitais e 5\% em hospices. Além disso, $66 \%$ atendem pacientes do Sistema Único de Saúde (SUS). Fatos esses que permitem a compreensão do panorama de evolução dos CP no país, que é recente e possui grande desigualdade em sua disponibilização entre as regiões do Brasil (Pinto, Harzheim, Hauser, D’Avila, Gonçalves, Travassos \& Pessanha, 2017).

Tratando-se da legislação brasileira, a PORTARIA Nº 483, DE $1^{\circ}$ DE ABRIL DE 2014 é uma das poucas existentes que aborda o assunto dos cuidados paliativos. Ela promete aos estabelecimentos que prestam atendimento a pessoas com doenças crônicas recursos, infraestrutura, qualificação de profissionais e diversos outros pontos importantes para sua consolidação (BRASIL, 2014). Como também, a RESOLUÇÃO No 41, DE 31 DE OUTUBRO DE 2018, que dispõe de diretrizes para a organização dos CP no âmbito do SUS, constando de indispensáveis normativas para o funcionamento adequado dos cuidados continuados. Tais quais: critério de elegibilidade, objetivos, oferta de cuidado precoce, disponibilização de medicamentos, oferta de assistência domiciliar àquele restrito ao leito ou ao domicílio (Diário Oficial da União, 2018). Portanto, nota-se que o país possui normativas para delinear os serviços de $\mathrm{CP}$, apesar de faltar um melhor direcionamento por elas, gerando o problema da incipiente implementação extensiva e igualitária dos serviços para todas as populações (Savassi, 2016).

Analisando os dados supracitados observa-se que ainda falta muito para o Brasil ter um fornecimento adequado de cuidados paliativos, como acontece na Austrália, Nova Zelândia e Reino Unido. É possível inferir, ainda, a partir das classificações e análises feitas sobre esse serviço no país que, mesmo com a existência de portarias que incentivem a implantação e desenvolvimento dos $\mathrm{CP}$, as garantias presentes nelas não estão sendo concretizadas.

É notável, portanto, a necessidade de uma melhor estruturação e investimento na modalidade paliativa no país. A ANCP - Academia Nacional de Cuidados Paliativos - em 2018 sugere que seja criada uma política de saúde que estruture de forma específica o desenvolvimento desses cuidados, destacando a importância de uma Política Nacional que oriente, por exemplo, os gastos com o serviço. Além disso, a organização de medidas de acesso aos medicamentos mais necessários é tida como indispensável, bem como a educação em saúde, visando a maior conscientização a respeito desse tema, que ainda é novo para a população brasileira no geral, incluindo profissionais da saúde.

Dessa forma, sobre a falta de conhecimento a respeito desse serviço, foi realizada uma pesquisa qualitativa 
questionando a informação que as pessoas possuem sobre os CP. O estudo demonstrou que o conhecimento adquirido pelos participantes provinha mais de experiências pessoais e profissionais, sendo muito pouco da mídia. Esses participantes reconheceram que até hoje existem muitos mitos e tabus em torno da morte, e, para isso, sugeriram que iniciativas educacionais midiáticas oferecidas por pessoas com experiência no assunto seriam benéficas para difundir o trabalho dos CP e a sua importância (Queiroz, 2011).

\section{A complexidade das Favelas brasileiras e os $\mathbf{C P}$}

Na segunda metade do século XIX, a população urbana do Brasil aumentou vertiginosamente. As cidades cresceram sem planejamento, e as pessoas pobres tiveram que se aglomerar em construções coletivas e insalubres: os cortiços denominação que faz alusão às colmeias de abelha. Na virada para o século XX, a elite financeira e política do Brasil, motivada por ideias da Belle Époque francesa e da medicina higienista, promoveu inúmeros desalojamentos compulsórios em várias cidades do país. O povo, então, teve que buscar soluções por conta própria, a saber: na falta de outras opções a população de baixa renda, na maioria das cidades brasileiras, sobe os morros ou ocupa as áreas de mangues e alagados, pouco valorizadas pelo mercado fundiário incipiente, gerando o "problema" das favelas (vilas, mocambos, palafitas, malocas, invasões, baixadas etc) (Cardoso, 2008; Queiroz, 2011).

À vista disso, limitações estruturais, educacionais, de transporte, saúde e segurança são fatores rotineiros em favelas, e enfrentados por seus moradores há mais de um século (Jeanniton, 2016). Dentre essa população vulnerada, encontram-se pessoas em situação ainda mais delicada, que são aquelas que necessitam de assistência integral em saúde em sua residência. Essas pessoas apresentam alguma condição progressiva ameaçadora da continuidade de sua vida, e carecem de auxílio externo. No entanto, essa situação em saúde se traduz, quase sempre, em dificuldades para se locomover aos centros de atendimento médicos para receber a assistência necessária (Hawley, 2017).

Fornecer cuidado em saúde a essa população é indispensável, e deve-se levar em conta as complexidades sociais dessa comunidade. Assim, o peso financeiro que quaisquer implementações tenham deve ser levado em consideração para que o usufruidor dessas ações consiga utilizar o serviço de forma adequada, sem que necessite despender parte considerável de seu parco orçamento (Jeanniton, 2016). Não é factível, desse modo, assumir a possibilidade de que essas pessoas possuam um cuidador 24 horas por dia, seja ele privado ou familiar, como exigem os Serviços de Atenção Domiciliar (SAD). Fato que denota a inadequação desses sistemas àqueles que necessitam desse acesso, mas acabam excluídos por não se enquadrarem a exigências burocráticas (Pinto, Harzheim, Hauser, D’Avila, Gonçalves, Travassos \& Pessanha, 2017; Savassi, 2016).

Além do mais, não há pesquisas focadas em levantar informações a respeito de como os CP são praticados nas favelas do Brasil, o que amplia a exclusão desses moradores também para as publicações científicas. Todavia, foram encontrados estudos que analisam as diferenças da execução dos CP em lugares economicamente distintos (Mcllfatrick, Noble, McCorry, Roulston, Hasson, McLaughlin, Johnston, Rutherford, Payne, Kemohan, Kelly \& Craig, 2014).

Assim, se reconhece que a transposição dos resultados dessas pesquisas para as favelas brasileiras é incipiente, mas possui utilidade para uma reflexão inicial. Em países desenvolvidos, um estudo mostrou que as pessoas acometidas por câncer são melhor assistidas quanto a CP nas regiões urbanas comparado à área rural e hospitais comunitários, evidenciando a disparidade ao acesso das populações menos favorecidas financeiramente (Hawley, 2017). No Canadá, outro estudo demonstrou que pessoas pobres tinham pouco acesso a esse tipo de cuidado, levando a resultados desfavoráveis à sua saúde, e que os CP iniciados precocemente proporcionavam melhor qualidade de vida e longevidade (Huynh, Henrym \& Dosani, 2015). Isso denota a importância da assistência em saúde efetiva para a população vulnerada, a qual, se ressalta, carece também de pesquisas a respeito de como esse serviço é realizado em suas localidades, assim como de seus resultados. 


\section{Considerações Finais}

$\mathrm{O}$ pouco interesse em investir nos $\mathrm{CP}$ está associado à negação sobre a morte e às superstições que a envolvem. Por essa razão a prática em saúde ainda é focada em manter a vida, com pouca ênfase em tornar o processo de morte menos doloroso e sofrido para os envolvidos. Sendo assim, para que os cuidados paliativos ganhem espaço e relevância entre os profissionais da saúde é necessária uma mudança estrutural, financeira e cultural, colocando o processo de morte e o morrer como uma situação de atuação desses trabalhadores, no reconhecimento da mesma como processo natural da vida e não de fracasso ou desistência. Assim, é de vital importância a formulação e aplicação de políticas públicas que garantam o melhor acesso aos CP e tudo aquilo que cerca os seus processos - formação, estrutura, equipamentos, equipe, aquisição de opioides.

Em relação aos CP nas favelas brasileiras, partindo da premissa que essas comunidades possuem vulnerabilidades maximizadas graças ao contexto socioeconômico que estão inseridas, associada ao deficitário serviço de CP no país, produzem razoáveis indícios que as pessoas residentes em favelas brasileiras possuem um acesso aos $\mathrm{CP}$ ainda mais incipiente que a população em geral. Todavia, tal consideração emerge de inferências produzidas por associação de dados, pois não há estudos empíricos até o presente momento que investigaram a temática.

Nesse sentido, é um problema premente a carência de pesquisas sobre $\mathrm{CP}$ focadas nas favelas brasileiras. Essa lacuna é sobremaneira grave, afinal há uma demanda de indivíduos elegíveis aos $\mathrm{CP}$ residentes em favelas que sequer o quantitativo é conhecido, os serviços de saúde e a comunidade científica não conhece o número pessoas que necessitam desses cuidados, quiçá as necessidades específicas de cuidado que esse cenário impõe. Esta lacuna deve ser preenchida para incluir os moradores desses locais nas publicações científicas e, principalmente, levantar informações que possam subsidiar ações adequadas para as peculiaridades de cada realidade a fim de garantir a efetividade do serviço.

Como trabalhos futuros, espera-se desenvolver outros estudos enfatizando a importância dos cuidados paliativos e da humanização para todos os níveis de atenção à saúde, principalmente na atenção primária, em todos os cantos do país, auxiliando os estudantes e profissionais de saúde na área.

\section{Referências}

Academia Nacional de Cuidados Paliativos. (2018). Panorama dos Cuidados Paliativos no Brasil. Academia Nacional de Cuidados Paliativos.

BRASIL. (2014). Portaria $N^{\circ}$ 483, de $1^{\circ}$ de abril de 2014. Redefine a Rede de Atenção à Saúde das Pessoas com Doenças Crônicas no âmbito do Sistema Único de Saúde (SUS) e estabelece diretrizes para a organização das suas linhas de cuidado. Brasil: Ministério da Saúde.

Carmo, M. E., \& Guizardi, F. L. (2018). O conceito de vulnerabilidade e seus sentidos para as políticas públicas de saúde e assistência social. Cad Saúde Pública; 34(3).

Hawley, P. (2017). Barriers to Access to Palliative Care. Palliat Care Res Treat

Huynh, L., Henrym B., \& Dosani, N. (2015). Minding the gap: access to palliative care and the homeless. BMC Palliat Care; $14(1): 62$.

Instituto Brasileiro de Geografia e Estatística. (2010). Censo Demográfico 2010: aglomerados subnormais, informações territoriais. IBGE.

Instituto Brasileiro de Geografia e Estatística. (2020). Distância do Estabelecimento de Saúde de Atenção Primária mais próxima ao Aglomerado Subnormal. $I B G E$.

Jeanniton, L. (2016). Low-Cost Approaches to Improve Quality of Life \&amp; Access to Healthcare in Brazilian Favelas. Clin Soc Work Heal Interv.

McIlfatrick, S., Noble, H., McCorry, N. K., Roulston, A., Hasson, F., McLaughlin, D., Johnston, G., Rutherford, L., Payne, C., Kernohan, G., Kelly, S., \& Craig, A. (2014). Exploring public awareness and perceptions of palliative care: A qualitative study. Palliat Medicine; 28(3):273-80.

Nickel, L., Oliari, L. P., Vesco, S. N. P. D., \& Padilha, M. I. (2016). Research groups in palliative care: the brazilian reality from 1994 to 2014. Esc Anna Nery - Revista; 20(1).

Pinto, L. F., Harzheim, E., Hauser, L., D’Avila, O. P., Gonçalves, M. R., Travassos, P., \& Pessanha, R. (2017). A qualidade da Atenção Primária à Saúde na Rocinha - Rio de Janeiro, Brasil, na perspectiva dos cuidadores de crianças e dos usuários adultos. Cien Saude Colet; 22(3):771-81.

Resolução $n^{\circ} 41$, de 31 de outubro de 2018. Dispõe sobre as diretrizes para a organização dos cuidados paliativos, à luz dos cuidados continuados integrados, no âmbito Sistema Único de Saúde (SUS). Diário Oficial da União. 
Research, Society and Development, v. 10, n. 6, e55110616183, 2021

(CC BY 4.0) | ISSN 2525-3409 | DOI: http://dx.doi.org/10.33448/rsd-v10i6.16183

Ribeiro, J. R., \& Poles, K. (2019). Cuidados Paliativos: Prática dos Médicos da Estratégia Saúde da Família. Revista Brasil Educação Medicina; 43(3), 62-72.

Santos, C. E., Campos, L. S., Barros, N., Serafim, J. A., Klug, D., \& Cruz, R. P. (2019). Palliative care in Brasil: present and future. Revista Associação Medica Brasileira; 65(6):796-800.

Savassi, L. C. M. (2016). Os atuais desafios da Atenção Domiciliar na Atenção Primária à Saúde: uma análise na perspectiva do Sistema Único de Saúde. Rev Bras Med Família e Comunidade; 11(38):1-12.

Schramm, F. R. (2007). Proteger os vulnerados e não intervir aonde não se deve Protect people who have become vulnerable and do not intervene where there is no need. Revista Brasilera de Bioética; 3(3):377-89.

Souza, M. T., Silva, M. D., \& Carvalho, R. (2010). Integrative review: what is it? How to do it? Einstein; 8(1):102-6.

Victor, G. H. G. G. (2016). Cuidados Paliativos no Mundo. Revista Brasileira de Cancerologia.

World Health Organization (WHO). (2020). Definition of palliative care. 